LA-14392

Approved for public release;

distribution is unlimited.

TRIDENT Flyer Plate Impact Technique: Comparison to Gas Gun Plate Impact Technique 
Los Alamos National Laboratory, an affirmative action/ equal opportunity employer, is operated by Los Alamos National Security, LLC, for the National Nuclear Security Administration of the U.S. Department of Energy under contract DE-AC52-06NA25396.

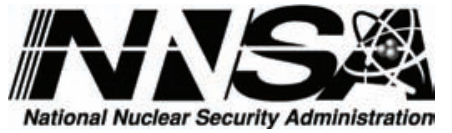

This report was prepared as an account of work sponsored by an agency of the U.S. Government. Neither Los Alamos National Security, LLC, the U.S. Government nor any agency thereof, nor any of their employees make any warranty, express or implied, or assume any legal liability or responsibility for the accuracy, completeness, or usefulness of any information, apparatus, product, or process disclosed, or represent that its use would not infringe privately owned rights. Reference herein to any specific commercial product, process, or service by trade name, trademark, manufacturer, or otherwise does not necessarily constitute or imply its endorsement, recommendation, or favoring by Los Alamos National Security, LLC, the U.S. Government, or any agency thereof. The views and opinions of authors expressed herein do not necessarily state or reflect those of Los Alamos National Security, LLC, the U.S. Government, or any agency thereof. Los Alamos National Laboratory strongly supports academic freedom and a researcher's right to publish; as an institution, however, the Laboratory does not endorse the viewpoint of a publication or guarantee its technical correctness. 
LA-14392

Issued: March 2009

\section{TRIDENT Flyer Plate Impact Technique:}

Comparison to Gas Gun Plate Impact Technique

Darcie D. Koller

George T. Gray III

Sheng-Nian Luo 
This page left blank intentionally. 


\title{
TRIDENT Flyer Plate Impact Technique: Comparison to Gas Gun Plate Impact Technique
}

\author{
Darcie D. Koller ${ }^{1}$, George T. Gray III ${ }^{2}$, and Sheng-Nian Luo ${ }^{3}$ \\ Los Alamos National Laboratory \\ ${ }^{1}$ Dynamic Experimentation and Materials Dynamics Division (DE-9) \\ ${ }^{2}$ Materials Science and Technology Division (MST-8) \\ ${ }^{3}$ Physics Division $(P-24)$
}

\begin{abstract}
This report describes the details of a series of plate impact experiments that were conducted on a gas gun in an effort to validate a new technique for plate impact using the TRIDENT laser to launch thin flyers. The diagnostics fielded were VISAR and identical samples and impactors were used on both platforms. All experimenters agree that the VISAR results should have agreed between the two experimental platforms. The VISAR results did not agree across the platforms and experimenters offer explanations and implications for this outcome.
\end{abstract}

\section{Background}

A series of plate impact experiments were conducted on a gas gun in an effort to validate a relatively new technique using the TRIDENT laser to launch flyers for plate impact experiments. Validation of the TRIDENT flyer plate impact technique is accomplished by comparing velocimetry data for these experiments to data from identical experiments (identical samples and geometries) carried out using the well established gas gun plate impact technique. The following questions are addressed in this study:

1. Do velocimetry results agree when experiments are carried out using identical impactors (same material, same geometry), impactor velocities and target materials? Are the apparent spall strengths observed identical regardless of experimental platform?

2. Is bulk response being observed with this experimental geometry?

3. Are the dynamic damage studies and techniques consistent with work accomplished on other experimental platforms?

\section{Results}

Table 1. summarizes the results of all experiments considered in this study. All experiments using TRIDENT were performed and analyzed by Shengnian Luo, and all experiments using the gas gun were performed and analyzed by Darcie Koller. Comments were solicited from George Gray III as a third party customer for the shock recovered samples and their data. 
Table 1 Experimental Details

\begin{tabular}{lccc}
\hline Shot ID & Platform & $\begin{array}{c}\text { Impactor velocity } \\
(\mathbf{m m} / \text { us })\end{array}$ & window \\
\hline $56-08-32$ & Gas gun & 0.177 & PMMA \\
19839 & TRIDENT & 0.188 & PMMA \\
20311 & TRIDENT & 0.220 & PMMA \\
$56-08-45$ & Gas gun & 0.226 & PMMA \\
20386 & TRIDENT & 0.143 & none \\
$56-08-50$ & Gas gun & 0.121 & none \\
$56-08-48$ & Gas gun & 0.085 & none \\
\hline
\end{tabular}

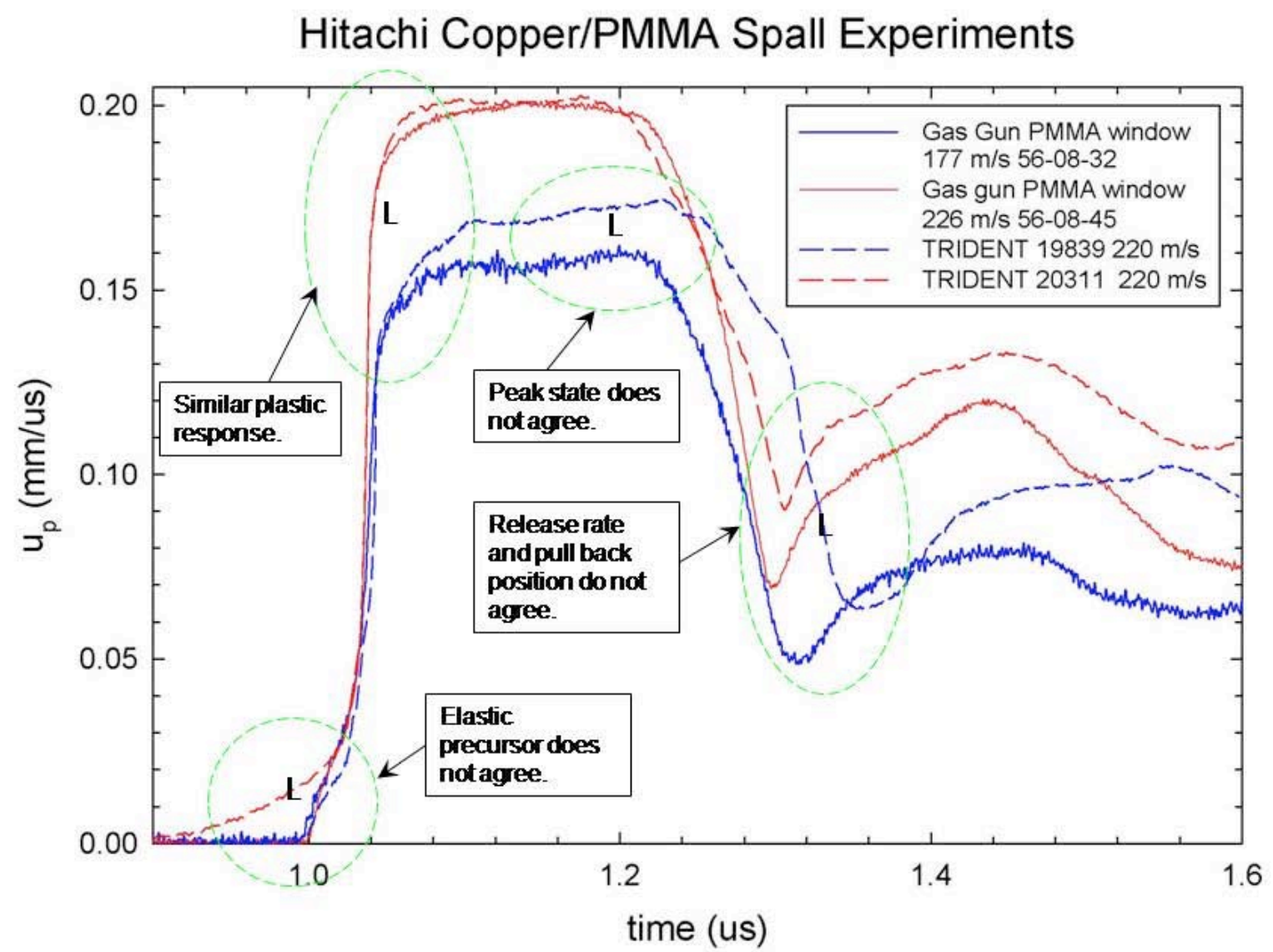

Figure 1 Overplot of velocimetry data from two TRIDENT laser launched flyer experiments at $\sim 220 \mathrm{~m} / \mathrm{s}$ with two gas gun experiments at $177 \mathrm{~m} / \mathrm{s}$ and $226 \mathrm{~m} / \mathrm{s}$. 


\section{Hitachi Copper Free Surface Spall Experiments}

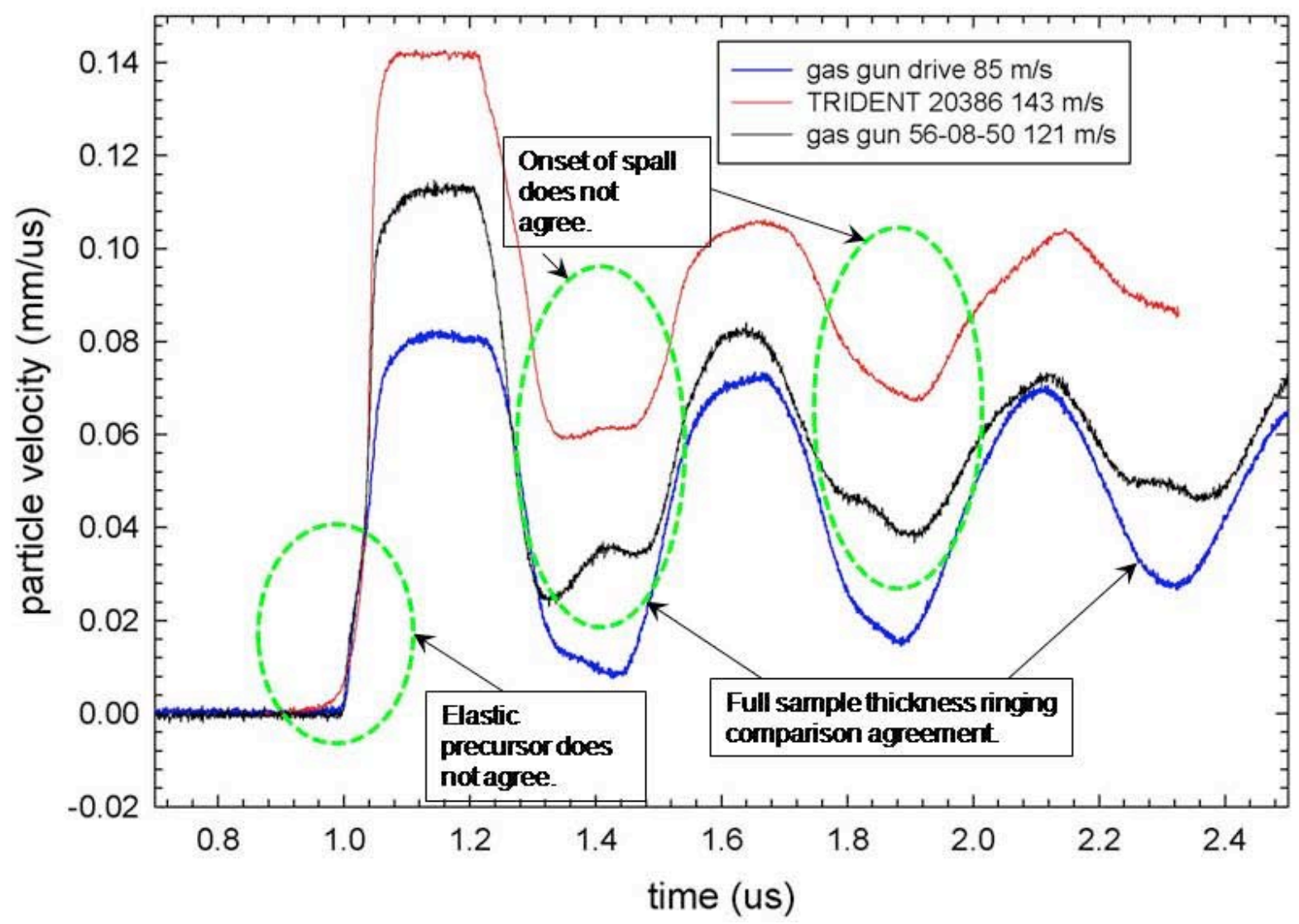

Figure 2 Overplot of velocimetry data from one TRIDENT free surface experiment with two free surface gas gun experiments.

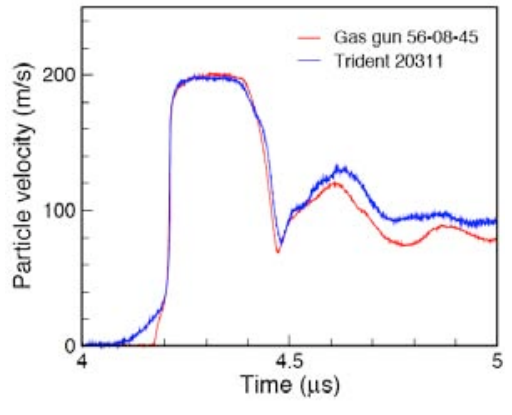

(a)

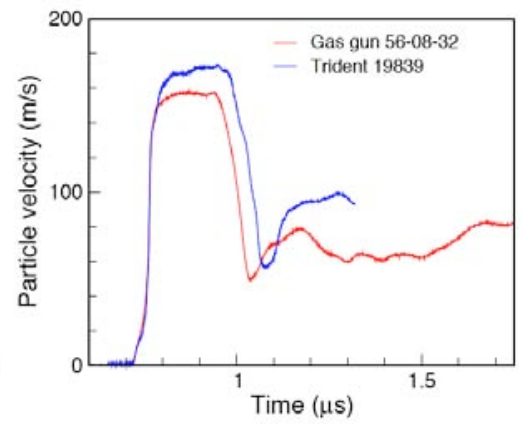

(b)

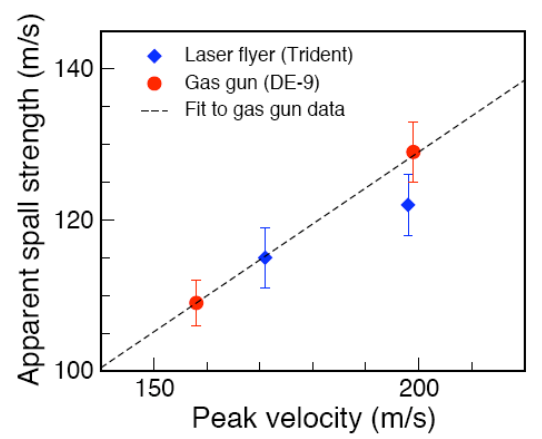

(c)

Figure 3 Overplot of the gas gun velocimetry data with the reprocessed TRIDENT velocimetry data for the PMMA window shots (reprocessed by Luo to address discrepancies with gas gun results) ( $a, b)$ and the plot of apparent spall strength vs. peak shock velocity (c). 


\section{Conclusions - Koller}

- Discrepancies cannot be explained by differences in experimental platform, with the exception of blowby issues due to laser launch technique.

- Discrepancies are a direct result of VISAR diagnostic execution and subsequent data analysis

- A possible solution to this problem is to require a validation diagnostic such as photon doppler velocimetry (PDV) to either validate or replace VISAR.

- These discrepancies yield misleading information about the spall response and plasticity of any material used in the TRIDENT flyer plate impact technique.

- The calculation of apparent spall strength is dependent on the slope of the release, depth of release before yielding begins, and slope of the pullback signal. Therefore, a similar calculated result can be reached even when velocimetry is in stark disagreement.

- Velocimetry will yield a unique and repeatable result if sample geometry, impact velocity, and sample material are kept constant.

- For the purposes of Hugoniot measurement this sample geometry provides enough of a steady state to lead to a Hugoniot measurement assuming accurate velocimetry results can be made.

- The caveat to this is that this is not an ideal technique for making Hugoniot measurements. A minimum of 2 experimental quantities (of the 5 quantities: $E, P, \rho, u_{p}, U_{s}$ ) must be measured to obtain a Hugoniot point (particle velocity only is not sufficient). The accuracy of those measurements will determine the accuracy of the Hugoniot point.

- For the purposes of dynamic damage experiments the volume of material sampled in tension will be extremely small using this sample geometry and length scale considerations must be clearly understood.

- The result of sampling small volumes in tension in a dynamic damage experiment over a short pulse duration yields, among other things, small void volumes.

- Shock recovery experiments of any kind explore the end state of the material and do not explore early time histories of microstructural evolution such as nucleation and void growth. That will require an in situ technique that has yet to be developed.

\section{Conclusions - Gray}

- The discrepancies described in this comparison provide a clear message of the need to carefully choose which platforms are most attuned to probing particular physics and the operative temporal and spatial length scales that they entail. In particular, processes such as EOS and Hugoniot data, including phase transformations, appear as favorable avenues for further TRIDENT studies. A comparison of wave profiles in a phase transforming metal, such as Zr, cross-compared with gun data would be useful to validate this position.

- Vetting of the VISAR data reductions utilized suggest an immediate need to cross correlate the procedures used for reducing raw VISAR data. Joint peer review of the raw data by additional VISAR experts appears warranted to clarify these questions immediately. 
- Conversely, long spatial and temporally dominated process, such as damage evolution and fracture processes which must survey a sizable sample size and time frame, due to shockwave shape effects, need to be carefully examined to determine if TRIDENT can statistically probe the microstructure AND the defect distributions in bulk engineering materials. The suggestion to continue to examine this question using PDV diagnostics to provide validated data appears warranted. Baring careful PDV analysis to clarify the questions of VISAR data reduction presented, I am concerned with the statistics of any damage and/or fracture (spallation) studies as representing bulk materials given the rising wave of experimental studies demonstrating the DOMINANT effects of shock pulse duration and shockwave profile shape on spallation.

\section{Conclusions - Luo}

- There exists overall agreement in the velocity histories and in the apparent spall strength within experimental uncertainties (Fig. 3). The platform dependence in such measurements is not considerable.

- The discrepancy in the elastic precursors, e.g., in Fig. 3(a), is likely caused by that the plasma shield dragged the target washer since the flyer and target were held together. This can be eliminated by physically separating the flyer assembly and the target assembly. Our diagnostics show the plasma blowby occurs at a later time for the barrel lengths we used. The VISAR measurement execution and data analysis may also contribute to the discrepancies in the velocity histories. For example, the VISAR probe laser spot in TRIDENT experiments is smaller. All these discrepancies can be resolved (e.g. with PDV). The thick plasma shield used in Trident 19839 [Fig. 3(b)] stuck with the flyer, and thus increased the effective thickness of flyer and delayed the release.

- Bulk responses in Hugoniot state, plasticity and spall can be probed with current geometry, as long as the probed area is sufficiently large compared to grain size.

- Shock spall-recovery experiments in TRIDENT show void formation in the incipient to full spallation but with smaller final void volume compared to larger flyer-target sizes. Thus, such experiments as in TRIDENT bear the merit in probing early stages of nucleation and growth in particular when recovery examination is involved, and the unloading rate can be different from other geometries. Spall is rate-dependent. The spall strength (after window correction) is consistent with previous results over a range of strain rates (e.g., see T. Antoun et al., S p all F r a c tu re, Springer, New York, 2003 and D. L. Paisley et al., R \& 79, 023902, 2008). 
This page intentionally left blank. 
This report has been reproduced directly from the best available copy. It is available electronically on the Web (http://www.doe.gov/bridge).

Copies are available for sale to U.S. Department of Energy employees and contractors from:

Office of Scientific and Technical Information

P.O. Box 62

Oak Ridge, TN 37831

(865) 576-8401

Copies are available for sale to the public from: National Technical Information Service

U.S. Department of Commerce

5285 Port Royal Road

Springfield, VA 22161

(800) 553-6847 
- Los Alamos NATIONAL LABORATORY

EST.1943 